\title{
Detection Rate of Fractures by Triage Nurses Applying the Ottawa Foot Rule
}

\author{
Abdullah Cuneyt Hocagil (iD, Hilal Hocagil (iD) \\ Department of Emergency Medicine, Bulent Ecevit University School of Medicine, Zonguldak, Turkey
}

Cite this article as: Hocagil AC, Hocagil H. Detection Rate of Fractures by Triage Nurses Applying the Ottawa Foot Rule. Eurasian J Emerg Med. 2018; 17 (3): 118-21.

\section{Abstract}

Aim: The purpose of this study was to investigate the detection rate of fractures by triage nurses by applying the Ottawa foot rule.

Materials and Methods: This is a prospective observational validation study that was designed in a training and research hospital between January and December 2013 on 98 patients with isolated foot injury. After triage nurses were provided training on the Ottawa foot rule for 4 hours, they evaluated patients with foot trauma by applying the rule. Foot radiographs were obtained from all trauma patients who were evaluated in the triage. Radiographs were evaluated by an emergency medicine specialist and fractures were determined.

Results: Data collection procedures included the evaluation of 90 out of 98 patients who demonstrated one or more qualities of the Ottawa foot rule according to the trained nurses. The fracture prediction rate of the triage nurses using the Ottawa foot rule was found to be $14.4 \%$. The sensitivity of the "the inability to take four steps in the Emergency Department" was $100 \%$ and the specificity was $41.6 \%$. Of all patients, $69.2 \%$ with fractures were aged $<55$ years, while $30.8 \%$ were aged $\geq 55$ years.

Conclusion: This study revealed that triage nurses could successfully perform the Ottawa foot rule after a brief training. According to the Ottawa foot rule applied by the triage nurses, the "inability to take four steps in the Emergency Department" rule was found to be the most significant.

Keywords: Emergency department, Ottawa foot rule, foot trauma

\section{Introduction}

Emergency departments (EDs) face overcrowding, particularly during rush hours. One of the reasons for this overcrowding is the admission of minor trauma patients (1). Acute foot injuries are the primary reason for applying to the ED with minor trauma, but there is no fracture detected in most of the patients upon radiological visualization $(2,3)$. Despite the low incidence of fractures, the common clinical practice in most EDs is to evaluate these patients using radiography to avoid medicolegal problems and patients' dissatisfaction in addition to the real indications (4-7).

To overcome this problem, clinical decision rules for ordering diagnostic radiographs for in-patients with acute foot injuries have been developed and the Ottawa foot rule is one such rule. Stiell et al. (8) described the Ottawa foot rule as experiencing tender pain at the navicular or basis of the fifth metatarsus, in the middle foot, inability to take four steps due to not bearing weight on foot immediately after trauma or in the ED (8-10).

ORCID IDs of the authors: A.C.H. 0000-0002-1675-9754; H.H. 0000-0001-7314-752X.

Correspondence to: Hilal Hocagil e-mail: drhocagil@gmail.com 
The Ottawa foot rule can determine the possibility of fracture and reduce the number of negative radiographies (11). A prospective observational study determined that ED triage nurses can successfully apply this rule (12).

The primary objective of this study was to investigate the detection rate of fractures by triage nurses applying the Ottawa foot rule.

\section{Materials and Methods}

\section{Study design and setting}

This prospective observational study was designed in a tertiary care hospital between January 2013 and December 2013 on 98 patients with isolated foot injury except ankle. The study protocol was examined and approved by the ethics committee of Dr Lutfi Kirdar Kartal Training and Research Hospital.

\section{Selection of participants}

All patients admitted to the ED with the complaint of foot trauma were investigated for eligibility in the study. All patients aged $>10$ years with isolated foot injury sustained within 7 days were included in the study and provided written informed consent.

Patients who had been transferred from another hospital on diagnosis of fracture, pregnancy, altered mental state, making an application for the second time, multiple trauma patients, and patients with isolated skin injuries, such as burns, abrasions, lacerations, and puncture wounds were excluded from the study.

\section{Observer groups}

The triage nurses group consisted of four ED nurses. The median age for the triage nurses was 30 years, and the median duration of their clinical experience in the ED was 7 years. The triage nurses groups included two males and two females.

\section{Study protocol}

Triage nurses were provided training in the anatomy of foot, Ottawa foot rule, objective of the study, study protocol for foot examination, and a practice of Ottawa foot rule for 4 hours by an emergency medicine specialist. After an interactive and applied training, data including patients' age and sex were collected and Ottawa foot rule screening was performed.

Triage nurses evaluated patients by applying the Ottawa foot rule as experiencing tender pain at the navicular or basis of the fifth metatarsus in the middle foot and the inability to take four steps immediately after trauma or in the ED. They recommended foot radiographies in patients meeting any of the rules. Foot radiographies were performed for all patients $(n=98)$ admitted to the ED and evaluated in the triage with foot trauma. Patients and their radiographies were evaluated by an emergency medicine specialist and fractures were determined. The sensitivity and specificity of each components of the Ottawa foot rule were calculated.

\section{Statistical analysis}

Statistical analyses were performed using the IBM Statistical Package for Social Sciences (IBM SPSS Corp.; Armonk, NY, USA) 19.0 software. Descriptive statistics were expressed as frequency and percentage.
The Fisher exact and Chi-square tests were used to determine the difference between groups. A p value of $<0.05$ was considered statistically significant for all tests.

\section{Results}

Ninety-eight patients who had applied to the emergency service with the complaint of isolated foot trauma were evaluated by the trained nurses and underwent foot radiography. Ninety of 98 patients demonstrated one or more component of the Ottawa foot rule (Figure 1), and 13 patients (14.4\%) were identified with a fracture in the foot radiograph. At least one component of the Ottawa foot rule was positive in all patients who were identified with fracture in the foot radiograph. The Ottawa rule applied by nurses for diagnosing foot fracture had a $100 \%$ sensitivity and $10.4 \%$ specificity.

Of the 90 patients who demonstrated one or more components of the Ottawa foot rule, $64.4 \%(n=58)$ were males and $35.6 \%(n=32)$ were females. The average age was $30 \pm 15$ years (min. 10, max. 70). The fracture rate was observed in $15.6 \%$ of females and $13 \%$ of males.

While $69.2 \%(n=9)$ of the patients with fractures experienced tender pain on the navicular or basis of the fifth metatarsus, $30.8 \%(n=4)$ experienced no tender pain. In contrast, $61.0 \%(n=47)$ of the patients without fractures experienced tender pain on navicular or basis of the fifth metatarsus. The sensitivity of the tender pain on navicular or basis of the fifth metatarsus with fractures was $69.2 \%$ and the specificity was $39.0 \%$ (Table 1 ).

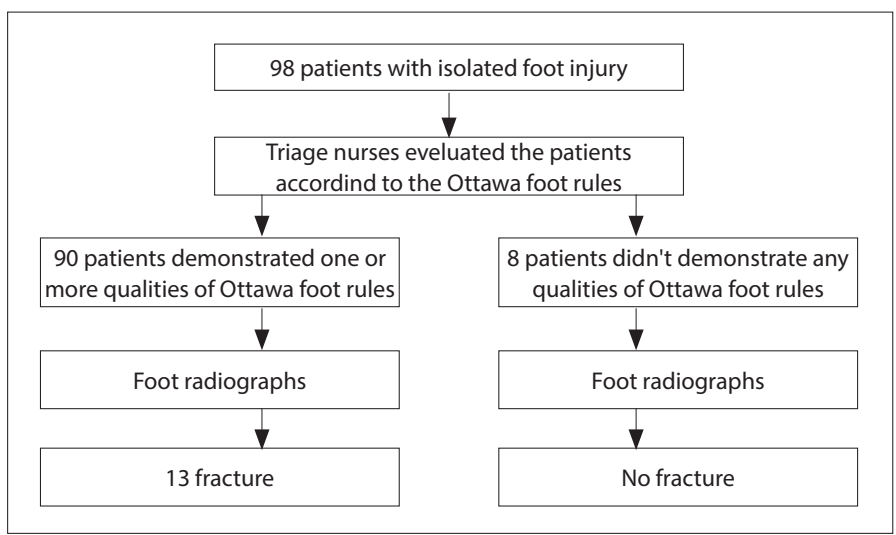

Figure 1. Flow diagram of the study population

Table 1. The relationship between Ottawa foot rule and fractures

\begin{tabular}{|l|c|c|c|c|}
\hline \multirow{2}{*}{\multicolumn{2}{|c|}{}} & \multicolumn{3}{|c|}{ Fracture } \\
\cline { 2 - 5 } & $\begin{array}{c}\text { No } \\
\%(\mathbf{n})\end{array}$ & $\begin{array}{c}\text { Yes } \\
\%(\mathbf{n})\end{array}$ & $\begin{array}{c}\text { Total } \\
\%(\mathbf{n})\end{array}$ \\
\hline Tender pain on navicular or & Yes & $69.2(9)$ & $61.0(47)$ & $62.2(56)$ \\
basis of the fifth metatarsus & No & $30.8(4)$ & $39.0(30)$ & $37.8(34)$ \\
\hline Tender pain on the & Yes & $46.2(6)$ & $81.8(63)$ & $76.7(69)$ \\
middle foot & No & $53.8(7)$ & $18.2(14)$ & $23.3(21)$ \\
\hline Inability to take & Yes & $100.0(13)$ & $58.4(45)$ & $64.4(58)$ \\
four steps & No & 0 & $41.6(32)$ & $35.6(32)$ \\
\hline
\end{tabular}


Table 2. Distribution of fractures according to age group

\begin{tabular}{|l|c|c|c|c|}
\hline \multicolumn{2}{|c|}{} & \multicolumn{2}{|c|}{ Age group, years } & \multirow{2}{*}{} \\
\cline { 3 - 4 } & $\begin{array}{c}\mathbf{< 5 5} \\
\mathbf{5}(\mathbf{n})\end{array}$ & $\begin{array}{c}\mathbf{> 5 5} \\
\mathbf{\%}(\mathbf{( n )}\end{array}$ & $\begin{array}{c}\text { Total } \\
\%(\mathbf{n})\end{array}$ \\
\hline \multirow{2}{*}{ Fracture } & Yes & $10.8(9)$ & $57.1(4)$ & $14.4(13)$ \\
\cline { 2 - 5 } & No & $89.2(74)$ & $42.9(3)$ & $85.6(77)$ \\
\hline Total & & $100.0(83)$ & $100.0(7)$ & $100.0(90)$ \\
\hline
\end{tabular}

While $46.2 \%(n=6)$ patients with a fracture felt tender pain in the middle foot, $53.8 \%(n=7)$ did not state any tender pain. Of the patients, $81.8 \%$ without a fracture felt tender pain in the middle foot. The sensitivity of tender pain on the middle foot among fracture cases was $46.2 \%$ and the specificity was $18.2 \%(p=0.010$; Table 1$)$.

Of the patients without fractures, $58.4 \%(n=45)$ were unable to take four steps in the ED. The sensitivity of the inability to take four steps in the ED for diagnosing a fracture was $100 \%$ and the specificity was $41.6 \%$ (Table 1).

Of all 90 patients, $7.8 \%(n=7)$ were aged $>55$ years. Fractures were identified in $10.84 \%(n=9)$ of patients younger than 55 years, while fractures were identified in $57.1 \%$ of patients older than 54 years $(p=0.008$; Table 2$)$.

\section{Discussion}

In this study, we aimed to evaluate the detection rate of fractures by triage nurses applying the Ottawa foot rule. We showed that the detection rate of fractures by triage nurses applying the Ottawa foot rule was $14.4 \%$. In recent studies, the detection rate of fractures by triage nurses was similar to that in our study. Crosswell et al. (6) performed a study on doctors and found the ratio to be $15 \%$. This ratio was found to be $16 \%$ and $17 \%$ in the studies conducted by Knudsen et al. (13) and Morrisin and Lovell (14). Our results were also similar to those of other studies and the ratio was $14.4 \%$. Hence, our nurses successfully performed the Ottawa foot rule.

Our nurses performed Ottawa foot rule after a 4-hour interactive and practical training, and we found that they could evaluate fracture with ratio of $14.4 \%$. Derksen et al. (15) found similar results where nurses could evaluate patients after a brief training on the Ottawa foot rule. In the United States, nurses who were working at the triage training were trained of the same practice to evaluate minor trauma patients, and they were provided the title of mid-level practitioners (16). This was performed to reduce overcrowding of the ED and workload of doctors. However, this practice was difficult due to the long and expensive training period, which was not a part of nursing (17).

In the present study, when the Ottawa foot rule was applied by the triage nurses, sensitivity and specificity of each parameter of the Ottawa foot rule was evaluated. Inability to take four steps in the ED was the best predictor within the rule. The sensitivity of this rule was $100 \%$ and the specificity was $41.6 \%$. Moreover, $100 \%$ sensitivity of this rule indicates the adequacy to suggest foot radiographies without considering other rules.
In this study, fracture incidence of patients $>55$ years was 5.28 times that of those $<55$ years. Hence, we can suggest that the Ottawa foot rule may be modified for two criteria, including the age of 55 years and the inability to take four steps immediately after trauma or in ED, similar to the Pittsburgh knee rule, which can be used as an alternative to the Ottawa knee rule, and includes criteria such as age and inability to take four steps immediately after trauma or in ED (18).

\section{Study limitations}

There exist certain limitations to this study. This is a single-center study, the number of patients was small, and the fracture rates were lower since major trauma patients were excluded.

\section{Conclusion}

Triage nurses could successfully perform the rule after a brief training. The similarity between doctors and triage nurses ratio for fracture determination with Ottawa rule is the indicator.

According to the Ottawa foot rule applied by the triage nurses, inability to take four steps in the ED was the most sensitive: the sensitivity of this criterion was $100 \%$ and the specificity was $41.6 \%$. Ottawa foot rule modified by the inability to take four steps in the ED and applied to the patients aged $>55$ years will be at the forefront and can reduce unnecessary imaging.

Ethics Committee Approval: Ethics committee approval was received for this study from the Ethics Committee of Dr Lutfi Kirdar Kartal Training and Research Hospital (Approval Date: 08.12.2012; Approval No.: 8951337/1009/136).

Informed Consent: Written informed consent was obtained from patients who participated in this study.

Peer-review: Externally peer-reviewed.

Author Contributions: Concept - H.H.; Design - H.H., A.C.H.; Supervision H.H., A.C.H.; Resources - H.H, A.C.H.; Materials - A.C.H.; Data Collection and/ or Processing - H.H.; Analysis and/or Interpretation - H.H., A.C.H.; Literature Search - H.H., A.C.H.; Writing Manuscript - H.H., A.C.H.; Critical Review - H.H., A.C.H.; Other - A.C.H.

Conflict of Interest: The authors have no conflict of interest to declare.

Financial Disclosure: The authors declared that this study has received no financial support.

\section{References}

1. Ganapathy S, Zwemer Jr FL. Coping with a crowded ED: an expanded unique role for midlevel providers. Am J Emerg Med. 2003; 21: 125-8. [CrossRef]

2. Emparanza Jl, Aginaga JR. Validation of the Ottawa Knee Rules. Ann Emerg Med. 2001; 38: 364-8. [CrossRef]

3. Roche A, Hunter L, Pocock N, Brown D. Physical examination of the foot and ankle by orthopaedic and accident and emergency clinicians. Injury. 2009; 40: 136-8. [CrossRef]

4. Whitely J, Goodacre S. Patient expectations of minor injury care: a cross-sectional survey Emerg Med J. 2014; 31: 567-70. [CrossRef]

5. Petscavage J, Baker SR, Clarkin K, Luk L. Overuse of concomitant foot radiographic series in patients sustaining minor ankle injuries. Emerg Radiol. 2010; 17: 261-5. [CrossRef] 
6. Crosswell S, Leaman A, Phung W. Minimising negative ankle and foot $X$-rays in the Emergency Department-Are the Ottawa ankle rules good enough? Injury. 2014; 45: 2002-4. [CrossRef]

7. Simon LV, Matteucci MJ, Tanen DA, Roos JA, Riffenburgh RH. J The Pittsburgh Decision Rule: triage nurse versus physician utilization in the emergency department. Emerg Med. 2006; 31: 247-50. [CrossRef]

8. Stiell IG, Wells GA, McDowell I, Greenberg GH, McKnight RD, Cwinn AA, et al. Use of radiography in acute knee injuries: need for clinical decision rules. Acad Emerg Med. 1995; 2: 966-73. [CrossRef]

9. Bachmann LM, Haberzeth S, Steurer J, ter Riet G. The accuracy of the Ottawa knee rule to rule out knee fractures. Ann Intern Med. 2004; 140: 121-4. [CrossRef]

10. Tay SY, Thoo FL, Sitoh YY, Seow E, Wong HP. The Ottawa Ankle Rules in Asia: validating a clinical decision rule for requesting $X$-rays in twisting ankle and foot injuries. J Emerg Med. 1999; 17: 945-7. [CrossRef]

11. Nichol G, Stiell IG, Wells GA, Juergensen LS, Laupacis A. An economic analysis of the Ottawa Knee Rule. Ann Emerg Med. 1999; 34:438-47. [CrossRef]

12. Szucs PA, Richman PB, Mandell M. Triage nurse application of the Ottawa Knee Rule. Acad Emerg Med. 2001; 8: 112-6. [CrossRef]
13. Knudsen R, Vijdea R, Dambourg F. Validation of the Ottawa ankle rules in a Danish Emergency Department Dan Med Bull. 2010; 57: 41-2.

14. Morris N, Lovell ME. Demographics of 3929 ankle injuries, seasonal variation in diagnosis and more fractures are diagnosed in winter. Injury. 2013; 44: 998-1001. [CrossRef]

15. Derksen R J, Bakker FC, Klerk ESML, Spaans IM, Heilbron EA, Veenings $B$, Haarman H JTM. Specialized emergency nurses treating ankle and foot injuries: a randomized controlled trial Am J Emerg Med. 2007; 25: 144-51. [CrossRef]

16. Dix A. Clinical management. Where medicine meets management. Let us play. Health Serv J. 2004; 114: 26-7.

17. Sakr M, Kendall R, Angus J, Sanders A, Nicholl J, Wardrope J, et al. Emergency nurse practitioners: a three part study in clinical and cost effectiveness. Emerg Med J. 2003; 20: 158-63. [CrossRef]

18. Cheung TC, Tank Y, Breederveld RS, Tuinebreijer WE, Klerk SML, Derksen RJ. Diagnostic accuracy and reproducibility of the Ottawa Knee Rule vs the Pittsburgh Decision Rule. Am J Emerg Med. 2013; 31: 641-5. [CrossRef] 